\title{
Maintaining Subject Engagement during Robotic Rehabilitation with a Minimal Assist-as-Needed (mAAN) Controller
}

\author{
Ali Utku Pehlivan, Dylan P. Losey Student Member, IEEE, Chad G. Rose Student Member, IEEE, \\ and Marcia K. O'Malley Senior Member, IEEE
}

\begin{abstract}
One challenge of robotic rehabilitation interventions is devising ways to encourage and maintain high levels of subject involvement over long duration therapy sessions. Assistas-needed controllers have been proposed which modulate robot intervention in movements based on measurements of subject involvement. This paper presents a minimal assist-asneeded controller, which modulates allowable error bounds and robot intervention based on sensorless force measurement accomplished through a nonlinear disturbance observer. While similar algorithms have been validated using healthy subjects, this paper presents a validation of the proposed mAAN control algorithm's ability to encourage user involvement with an impaired individual. User involvement is inferred from muscle activation, measured via surface electromyography (EMG). Experimental validation shows increased EMG muscle activation when using the proposed mAAN algorithm compared to nonadaptive algorithms.
\end{abstract}

\section{INTRODUCTION}

Robotic rehabilitation is a promising path towards implementing high intensity, long duration, and repetitive movement therapies for recovery after a neurological injury [1]. However, high levels of voluntary effort are also required for robotic rehabilitation to best facilitate neural plasticity and recovery [2], [3]. Towards this end, allowing movement errors can prevent subjects from becoming overly reliant on the robot, and "error" is therefore crucial in facilitating motor learning [4], [5]. Many methods for allowing movement error and maintaining subject engagement have been proposed; traditionally, impedance controllers have been used to relate forces with deviations from the robot's desired trajectory [6]. Alternatively, assist-as-needed (AAN) controllers both ensure that subjects successfully complete the desired task, and promote the subject's active, cognitive participation [7]. For instance, position-based adaptive AANs predict the human's capabilities as a function of workspace location [7], [8], and, more recently, sensorless force estimation techniques have been incorporated in AAN controllers to determine subject's capabilities in real time [9].

While there are several ways to establish the interaction between the robot and the patient during robotic rehabilitation [3], [5]-[9], not all of these techniques are optimal in the sense of maximizing patient engagement. In our previous study [9], we summarized the limitations of these robotic

D.P. Losey, C.G. Rose, and M.K. O'Malley are with the Department of Mechanical Engineering, Rice University, Houston, TX 77005 (corresponding email: cgr2@rice.edu).

This work was supported by NSF-CPS-1135949, NSF GRFP-1450681, NSTRF NNX13AM70H, and Mission Connect, a project of the TIRR Foundation.

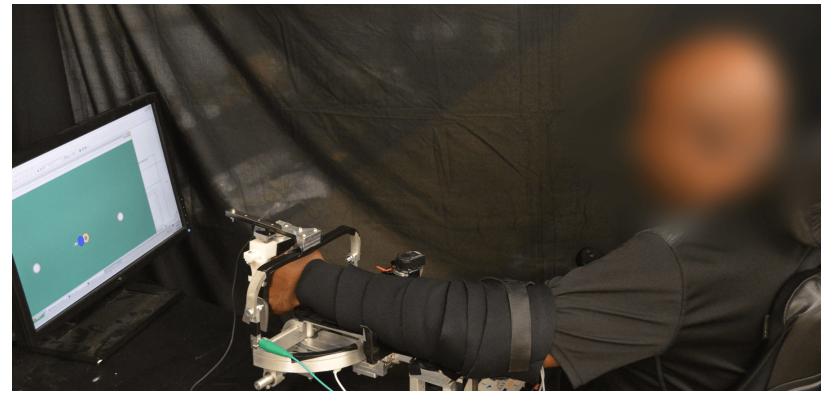

Fig. 1. Subject with incomplete SCI interacting with the RiceWrist-S We tested how a mAAN rehabilitation controller encouraged this subject's engagement during robotic rehabilitation as measured by surface EMG.

rehabilitation controllers, and proposed a minimal assist-asneeded (mAAN) controller which is capable of avoiding many of these limitations. In that study, we showed that mAAN approach more accurately estimates the subject's capabilities than position-dependent methods [7], [8], while also continually adapting assistance levels to the user's current ability, unlike traditional impedance controllers [6].

In this study, our aim is to evaluate the effectiveness of the mAAN paradigm through a case study with an incomplete spinal cord injury (SCI) subject (Fig. 1). We specifically test the paradigm's ability to maintain subject engagement, where engagement is inferred via EMG. Measuring EMG gives access to the subject's modulation of muscle activation during movements with the robot rather than inferring such activity through measurement of the resultant forces.

Previous work [9] developed a Kalman filter (KF) to estimate subject input and system states, but other techniques can fill this role. Here, we have formulated our mAAN controller using the nonlinear disturbance observer (NDO) proposed by Chen et al. [10]. The primary motivation behind employing this NDO is its disturbance estimation capability with a bounded estimation error even under time-varying disturbances. After formulating our rehabilitation setting in Section II, we introduce this NDO in Section III. Section IV presents the details of the proposed mAAN controller which incorporates this NDO. Section V describes the results of this single-subject study, and Section VI summarizes how mAAN controllers can encourage patient engagement.

\section{PROBLEM STATEMENT}

The experimental platform used in this study was the RiceWrist-S, a three degree-of-freedom (DOF) serial forearm-wrist exoskeleton. The device is capable of independently actuating forearm pronation/supination (PS), wrist 
flexion/extension (FE), and radial/ulnar deviation (RU). In a previous work [11] we demonstrated that the RiceWrist-S possesses mechanical capabilities which make it especially suitable for rehabilitation applications. The following model represents the dynamics of the RiceWrist-S:

$$
M(q) \ddot{q}+C(q, \dot{q}) \dot{q}+G(q)=\tau_{r}+\tau_{p}
$$

Here $q$ is a $3 \times 1$ vector of joint positions, $M$ is the $3 \times 3$ inertial matrix, $C$ is the $3 \times 3$ matrix which represents Coriolis/centrifugal terms, $G$ is the $3 \times 1$ gravity vector, $\tau_{r}$ is the $3 \times 1$ vector of torques applied by the actuators, and $\tau_{p}$ is the $3 \times 1$ vector of torques applied by the subject and mapped into joint space. The proposed mAAN controller was implemented on the FE joint of the manipulator; the unused DOFs were physically constrained. System parameters (static friction, inertia, and viscous friction) have already been experimentally identified for FE joint [11].

By "subject capability," we mean the input force which the subject applies at a given time. If the subject applies forces which move the robot along the desired trajectory, then they are capable of performing the task alone, and the robot will remain backdrivable within a defined error bound. If the subject applies forces which would cause the robot to significantly deviate from the desired trajectory, then the robot will intervene to provide assistance. Force sensors at the end-effector are an obvious solution for determining subject capability. The inclusion of force sensors has drawbacks, however, such as introducing stability concerns, the need for frequent calibration, and an increase in the system cost.

One cost-effective alternative is sensorless force estimation, a technique that exploits differences between the expected and actual manipulator configurations to approximate applied disturbances. In particular, in this work we implement a model-based sensorless force estimation approach, where plant dynamics and input-output data are used to extract the user's applied force. If the plant model is inaccurate, this disturbance estimation method cannot correctly distinguish between responses caused by known and unknown inputs; the estimated disturbances therefore include both external forces $\left(\tau_{p}\right)$ and unmodeled dynamics $\left(\tau_{m}\right)$. While this effect may seem undesirable, we show that model-based disturbance estimation can still be quite convenient for robotic rehabilitation applications. For the sake of formulation, let us denote the disturbance which our model-based approach seeks to estimate as

$$
d=\tau_{p}+\tau_{m}
$$

Applying the disturbance definition (2), the robot manipulator dynamics (1) can be rewritten

$$
\hat{M}(q) \ddot{q}+\hat{C}(q, \dot{q}) \dot{q}+\hat{g}(q)=\tau_{r}+d
$$

where $(\hat{\cdot})$ donates an estimated value.

\section{NONLINEAR DISTURBANCE OBSERVER}

The NDO originally proposed by Chen et al. [10] has been employed in a variety of applications, such as friction compensation, sensorless torque control and haptic interaction control [12]. In this section we will provide an overview of the disturbance observer formulation from [10]. As an additional step, we further show that disturbance estimation errors are bounded in the case of a time-varying interaction between the subject and robotic device. First, the NDO formulation defines a differential equation-which describes the disturbance estimation system-as follows

$$
\dot{\hat{d}}=-L(q, \dot{q})(\hat{d}-d)
$$

Here $\hat{d}$ is the estimate of the disturbance term, and $L(q, \dot{q})$ is defined with the following relation

$$
L(q, \dot{q}) \hat{M}(q) \ddot{q}=\frac{d p(q, \dot{q})}{d t}
$$

where $p(q, \dot{q})$ will be determined subsequently. The main purpose of this disturbance observer formulation is to eliminate the need for acceleration measurement via the inclusion of an auxiliary variable $z$

$$
z=\hat{d}-p(q, \dot{q})
$$

Considering the relations given in (4-6), and the robot manipulator dynamics given in (1), the time derivative of this auxiliary variable $z$ is as follows

$$
\begin{gathered}
\dot{z}=-L(q, \dot{q})(z+p(q, \dot{q}))+L(q, \dot{q})(\hat{M}(q) \ddot{q}(t)+\hat{C}(q, \dot{q}) \dot{q}(t) \\
\left.+\hat{g}(q)-\tau_{r}(t)\right)-L(q, \dot{q}) \hat{M}(q) \ddot{q} \\
=-L(q, \dot{q})\left(z+p(q, \dot{q})-\hat{C}(q, \dot{q}) \dot{q}-\hat{g}(q)+\tau_{r}\right)
\end{gathered}
$$

Note that the above relation allows computation of the auxiliary variable $z$, and hence the estimation of the disturbance $\hat{d}$, without acceleration information. Of course, in order to achieve this, the functions $L(q, \dot{q})$ and $p(q, \dot{q})$ have to be designed such that the disturbance estimation term asymptotically converges to its true value. Towards this aim, let us define the disturbance estimation error term such that

$$
e_{d}=d-\hat{d}
$$

Within this setting, disturbances are typically assumed to be constant, i.e., $\dot{d}=0$, noting that our estimation method will still be shown to work even when this assumption is strongly violated. Now, recalling the relation given in (4), the time derivative of the error term can be written

$$
\dot{e}_{d}=-L(q, \dot{q}) e_{d}
$$

By inspecting (9), we see that the proper selection of $L(q, \dot{q})$ will cause $e_{d}$ to asymptotically converge to zero. Considering (5), the selection of $p(q, \dot{q})=c \dot{q}$ (where $c$ is a positive constant) gives the following relation

$$
L(q, \dot{q})=c \hat{M}^{-1}(q)
$$

Due to the inherent properties of the inertia matrix for robotic manipulators (symmetry and positive definiteness), the selection of $L(q, \dot{q})$ in (10) desirably leads to asymptotic convergence of the error term in (9). 


\section{A. Existence of Time Varying Disturbances}

In a rehabilitation application, the force input (subject capability) changes as a function of time, and, clearly, our disturbance estimation technique should accurately account for these changes. When time varying disturbances exist, the assumption $\dot{d}=0$ will not be correct. Hence, the disturbance estimation error system (9) can be redefined as follows

$$
\dot{e}_{d}=-L(q, \dot{q}) e_{d}+\dot{d}
$$

There does not exist an equilibrium point for the error system in (11). Hence the disturbance estimation error system is not stable in the sense of Lyapunov. Lyapunov stability analysis, however, can be used to show the boundedness of the solutions [13], so that our estimation errors have a limited magnitude. We employ the following Lyapunov function candidate when $\dot{d} \neq 0$

$$
V_{o, N D O}\left(e_{d}\right)=\frac{1}{2} e_{d}^{T} e_{d}
$$

We here assume that the time derivative of the user input is upper bounded with a constant $D$, where $\|\dot{d}\| \leq D$. This assumption is reasonable since subjects capability for providing input is limited in terms of movement frequency. Note that the $L_{2}$ norm is employed throughout the formulation for consistency. Now, substituting in (11) and (10), the time derivative of the Lyapunov function yields

$$
\begin{aligned}
& \dot{V}_{o, N D O}\left(e_{d}\right)=-e_{d}^{T}\left(c \hat{M}^{-1}\right)(q) e_{d}+\dot{d} \\
& \leq-c \lambda_{\min }(\hat{M})\left\|e_{d}\right\|^{2}+D\left\|e_{d}\right\|
\end{aligned}
$$

We used the relation $y^{T} x \leq\|x\|\|y\|$ in the above inequality, where $\lambda_{\min }$ is the minimum eigenvalue of the inertia matrix $M$. By introducing a constant $\theta$, such that $0<\theta<1$, (13) can be represented in the following form

$$
\begin{gathered}
\dot{V}_{o, N D O}\left(e_{d}\right) \leq-(1-\theta) c \lambda_{\min }(\hat{M})\left\|e_{d}\right\|^{2}+ \\
\theta c \lambda_{\min }(\hat{M})\left\|e_{d}\right\|^{2}+D\left\|e_{d}\right\|
\end{gathered}
$$

So, we conclude $\dot{V}_{O, N D O} \leq-(1-\theta) c \lambda_{\min }(\hat{M})\left\|e_{d}\right\|^{2} \forall e_{d}$ if and only if

$$
\left\|e_{d}\right\|>\frac{D}{\theta c \lambda_{\min }(\hat{M})}
$$

Therefore, as soon as the inequality in (15) is satisfied, the error system will behave like it is asymptotically converging to the equilibrium point-i.e., like the case where $\dot{d}=0$. It is possible to define an ultimate bound on the disturbance estimation error $e_{d}$ with a more rigorous analysis, but, for the sake of briefness, we use a constant $e_{U B}$ as an upper bound on right side of (15). The performance of the described NDO for a time varying disturbance is shown in Fig. 2; we note that the estimation of subject capabilities remains accurate, even when the subject's applied force is rapidly changing.

\section{MAAN CONTROL LAW}

Since the proposed NDO is robust to time-changing subject capabilities, we next incorporate it within our mAAN controller. In this section, we will focus on the mAAN control law, noting that the associated stability analysis

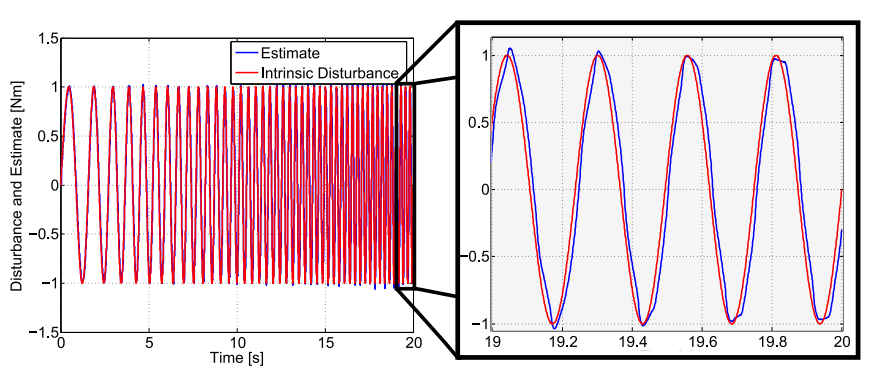

Fig. 2. Experimental validation of the disturbance estimation capability of our NDO. The applied torque is a chirp signal sweeping from $0.5 \mathrm{~Hz}$ to 4 $\mathrm{Hz}$ over $20 \mathrm{~s}$ with $1 \mathrm{Nm}$ amplitude. The maximum disturbance estimation error is less than $16 \%$ of the disturbance's amplitude at any given time.

has already been performed with the KF method [9], and thus does not need to be repeated. An AAN controller for rehabilitation exercises should help subjects complete desired motions while encouraging active participation. Accordingly, the following mAAN controller is introduced

$$
\tau_{r}=\tau_{b}-\hat{d}
$$

where $\tau_{b}$ signifies a baseline controller, $\hat{d}$ indicates the model-based disturbance estimate (from the NDO), and $\tau_{r}$ represents the robot's joint torques. This AAN controller has the same structure as those in [7], [8], and [9]. Intuitively, whenever the human provides an input to backdrive the robot along the desired trajectory, $\tau_{b}-\hat{d}=0$, and so the robot provides no assistance. By constrast, when subjects make mistakes, (16) works to compensate for their applied disturbance. Because of the bounded errors within the NDO, (15), some tracking error is inevitable; we will subsequently demonstrate that this tracking error can beneficially motivate participation during training.

The baseline controller, $\tau_{b}$, is selected to be the passivitybased motion control law proposed by [14] and detailed in [15]. With a twice-differentiable reference trajectory, we express the position error in joint space as $\tilde{q}=q-q^{d}$. We then define the desired motion variables

$$
v=\dot{q}^{d}-\Lambda \tilde{q} ; \quad a=\dot{v} ; \quad r=\dot{q}-v
$$

where $\Lambda$ is a positive definite matrix which determines the relative weight of position errors. The baseline controller can then be written

$$
\tau_{b}=\hat{M}(q) a+\hat{C}(q, \dot{q}) v+\hat{g}(q)-K_{D} r
$$

where $K_{D}$ is a positive definite gain matrix. In [9], we extensively studied the stability of (1), (16), and (18) when $\hat{d}$ was determined using a KF approach. Because these derivations are almost identical when the NDO from Section III is used instead of this KF approach, we will here skip to the conclusion of this stability analysis, referring the interested reader to [9] for more information. An important point to consider however is that the stability analysis of the mAAN controller, (16), employing our NDO, $\hat{d}$, yields bounded trajectory errors (see Fig. 3); bound being governed by the following equation

$$
\|r\| \geq \frac{\left\|\tilde{M} r+\tilde{C} r-e_{U B}\right\|}{\epsilon \lambda_{\min }\left(K_{D}\right)}
$$




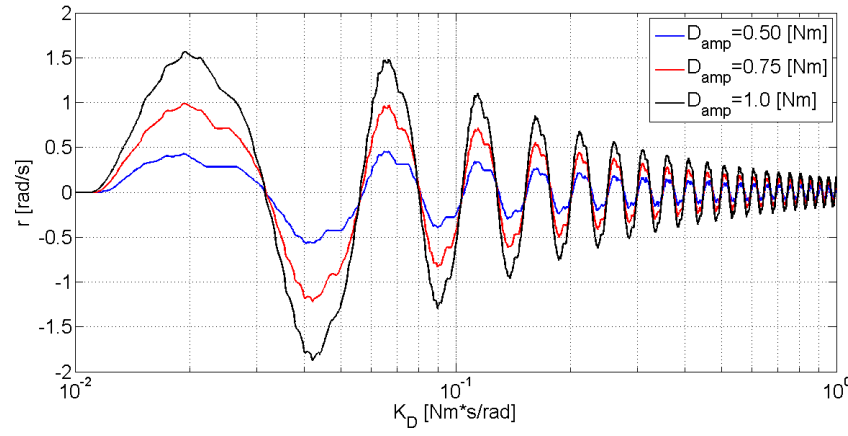

Fig. 3. Trajectory error using mAAN control and an NDO with both varying $K_{D}$ values and disturbance amplitudes. Applied torques were $1 \mathrm{hz}$ sinusoidal with amplitude $D_{a m p}$. The robot was commanded to maintain a stationary pose. As $K_{D}$ increases the error bounds tighten; here the amount of error $r$ resulting from an identical input decreases in response to increased $K_{D}$. Disturbances with greater amplitude desirably create larger errors.

where $\epsilon$ is a constant, such that $0<\epsilon<1$. Importantly, (19) ensures that the human is able to make errors during movements, but the magnitude of the error can be bounded by changing the user-selected gain matrix $K_{D}$. We will next discuss an algorithm which automatically modulates $K_{D}$, and therefore the magnitude of the subject's allowable error.

\section{A. Error Bound Modification Algorithm}

Our mAAN controller with an NDO ensures that subjects complete the movement task while providing minimal assistance. In order to further encourage active participation, however, it is necessary to properly "challenge" the subject. If the subjects are not challenged, they may become passive and let the robot complete their movements [16]. With (19) we have shown that the movement errors are bounded; intuitively, we would like to allow less impaired subjects to make greater errors (and therefore have a more challenging task), while severely impaired subjects should only be able to make small errors (making the task suitably easy). As we have pointed out, changing the matrix $K_{D}$ modulates the amount of allowable error. Like in [9], we thus introduce an algorithm to automatically update $K_{D}$ in order to maintain a user-specified allowable trajectory error:

$$
K_{D, i+1}=\left(1+x_{i}\right) K_{D, i}
$$

The feedback gain for the next task, $K_{D, i+1}$, is determined by comparing the subjects' current average error, $\bar{r}_{i}$, to the maximum allowable average error, $r^{*}$. As such, $x_{i}$ in (20) can be formulated as:

$$
x_{i}=x_{n o m} \frac{\bar{r}_{i}-r^{*}}{r^{*}}\left(\frac{\left|\bar{r}_{i}-r^{*}\right|}{\left|\bar{r}_{i-1}-r^{*}\right|}\right)^{\operatorname{sign}\left(r^{*}-\bar{r}_{i}\right)}
$$

Here $x_{n o m}$ is a predetermined, constant, nominal change rate. The sign of $x_{i}$ is determined by comparing the average error in the current task to the maximum allowable error. To better understand how this algorithm works in practice, we refer the reader to [9], where the authors performed initial healthy-subject experiments. In this paper, we will experimentally use this error bound modification algorithm and mAAN controller to encourage the participation of an SCI subject, as described in the next section.

\section{EMG-BASED EXPERIMENTAL VALIDATION}

The main purpose of the proposed subject adaptive algorithm is to maintain subject engagement throughout training. We experimentally examined how the inclusion of the proposed mAAN controller would affect the variation in subject involvement during wrist flexion/extension movements. A four-session robotic training protocol was conducted with a single subject, a 47 year-old male with incomplete SCI at the C3-5 level, American Spinal Injury Impairment Scale (AIS) C. The four sessions took place on spearate days spanning a two week period. Each session consisted of two separate training blocks $\mathrm{a}$ and $\mathrm{b}$ (training blocks are referred subsequently by using the associated session numbers as (session no)-a and (session no)-b). A given training block consisted of two ten minute phases, separated by a short break. The subject adaptive algorithms were not included in the first phase, but were implemented with the AAN controller in the second phase.

Throughout the training, visual feedback, which is described in Fig. 4, was presented to the subject. The allocated time to move from center-to-periphery targets was one second, the targets at the periphery were placed at $\pm 20^{\circ}$, and the targets were presented randomly. The subject was instructed to follow the given desired trajectory as close as possible; however, intentional movements faster than given desired trajectory were not discouraged. For the subject adaptive algorithms, the initial, minimum, and maximum feedback gains were assigned to be $10^{-2}, 10^{-5}$, and 0.5 $\mathrm{Nm} \cdot \mathrm{s} / \mathrm{rad}$, respectively, and the $r^{*}$ value was set to 0.040 $\mathrm{rad} / \mathrm{s}$. For the case where AAN was implemented without subject adaptive algorithms the feedback gain was assigned as $0.5 \mathrm{Nm} \cdot \mathrm{s} / \mathrm{rad}$.

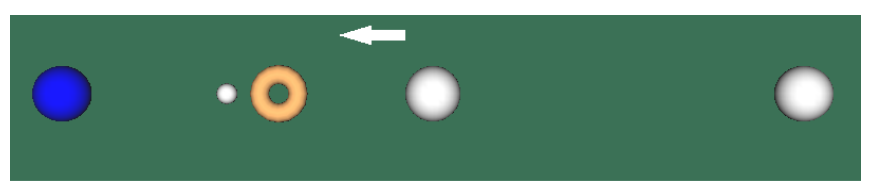

Fig. 4. Visualization used for human-robot experiments. The torus (orange) represents the subject's position, the white ball indicates desired position, and the current target is highlighted in blue. A task consisted of moving from the center to one of the peripheral targets along the desired trajectory.

The effect of the subject adaptive algorithms on participant engagement in therapy was evaluated by measuring muscle activity with surface electrodes on the arm, similar to the methods by Krisnan et al. [17], during trials with and without the algorithms active. Measurements were taking during only the first and second ten minute phases for a given training block to minimize the effect of fatigue.

Since the experiment was implemented on the FE joint of the RiceWrist-S, the muscles of interest were flexor carpi radialis (FCR) and extensor carpi ulnaris (ECU). A Delsys Bagnoli-8 surface EMG system was used to collect EMG data. Skin preparation was conducted according to [18], using fine sand paper along with isopropyl alcohol wipes 
prior to electrode placement. The electrodes were placed approximately over the bellies of the FCR and ECU muscles. To reduce possible electromagnetic interference (EMI) caused mainly by electrical actuators, the conductive parts of the exoskeletal frame were grounded and the subject's arm was wrapped in neoprene. Additionally, in order to decrease any possible crosstalk between FCR and ECU muscles, and finger muscles due to grip force a special handle was used, which can be coupled to the palm of the subject so that subject does not need to grip during the training. A $1000 x$ gain was chosen on the Delsys system to amplify the voltage reading, and the system provided a $20-450 \mathrm{~Hz}$ band-pass filter. The data was recorded with a QuaRC (Quanser Inc.) data acquisition system at a sampling rate of $1 \mathrm{KHz}$. Another filtering stage was applied digitally using a $25-450 \mathrm{~Hz}$ bandpass discrete filter to reduce EMI. As a final step, the magnitude of the band-pass filtered EMG data was smoothed for the analysis using a $100 \mathrm{~ms}$ window running root mean square (RMS) calculation.

Although the aim was to use the data collected from both the FCR and ECU muscles, ECU muscle measurements were unreliable, arising from a combination of the subjects inability to activate the ECU muscle, and a thick skin layer on the outer forearm. Hence the following data analysis is conducted by using FCR muscle data only when the desired movement was in the flexion direction. The subject involvement was calculated as a percentage of time, using the ratio between involvement time, $t_{i n v}$, and the total time, $t_{\text {total }}$. The involvement time, $t_{\text {inv }}$, was calculated as the amount of time the RMS value of the EMG data was larger than a threshold of the maximum voluntary contraction (MVC) recorded on a particular session. For the purposes of analysis in this study, the threshold was set to 0.20 . The total time, $t_{\text {total }}$, was calculated as the amount of time for the subject to reach the desired target for a flexion movement. Since the implemented subject adaptive algorithms allowed intentional movement faster than the desired movement, the amount of time for the subject to reach the desired target was used in the calculation of $t_{\text {total }}$, rather than the complete allocated time for the desired movement, which was one second as specified previously.

The subject involvement performances, for the cases with and without the inclusion of the subject adaptive algorithms within the AAN controller, were compared using the data gathered throughout the eight training blocks. Fig. 5 shows the average subject involvement values for each 50 second interval of a given training block, labeled, for example, as 1-a (session 1, training block a). The results reveal that for 6 out of 8 training blocks, average subject involvement was consistently higher for training blocks when the subject adaptive algorithms were active. For the training blocks 2-a and 4-b, average subject involvement was similar for both cases. These findings are supported by Fig. 6(a) which presents the average percentage of time across trials for each block. Again, it is noted that, with the exception of training blocks 2-a and 4-b, subjects involvement as measured by EMG is greater when the adaptive algorithms are active.
An important trend was observed when the presented average subject involvement values in Fig. 5 were averaged over all the training blocks. Fig. 6(b) presents that, for the case without the inclusion of the subject adaptive algorithms the average subject involvement shows an apparent decreasing

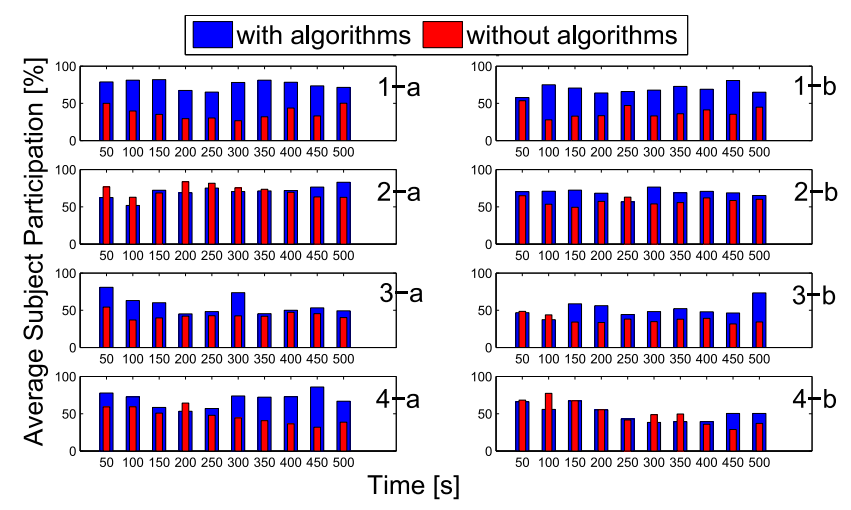

Fig. 5. The subject involvement performances for the cases with and without the inclusion of the subject adaptive algorithms within the mAAN controller shown for every training block separately. In 6 out of 8 training blocks, average subject involvement was consistently higher for the case with the inclusion of the subject adaptive algorithms.

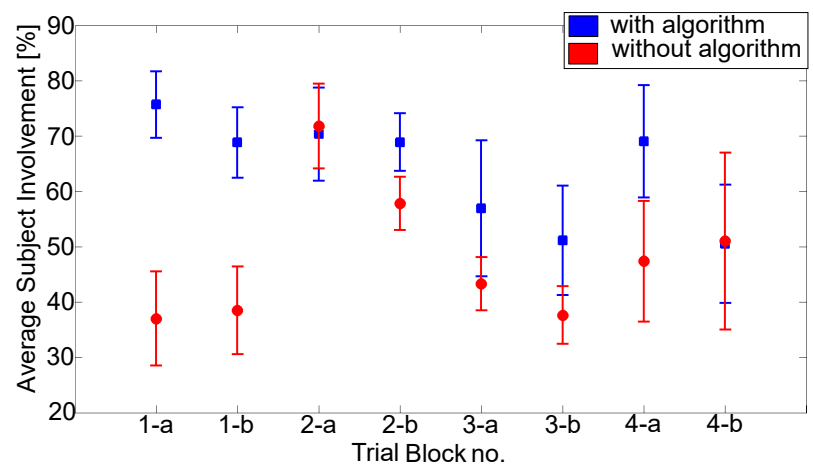

(a)

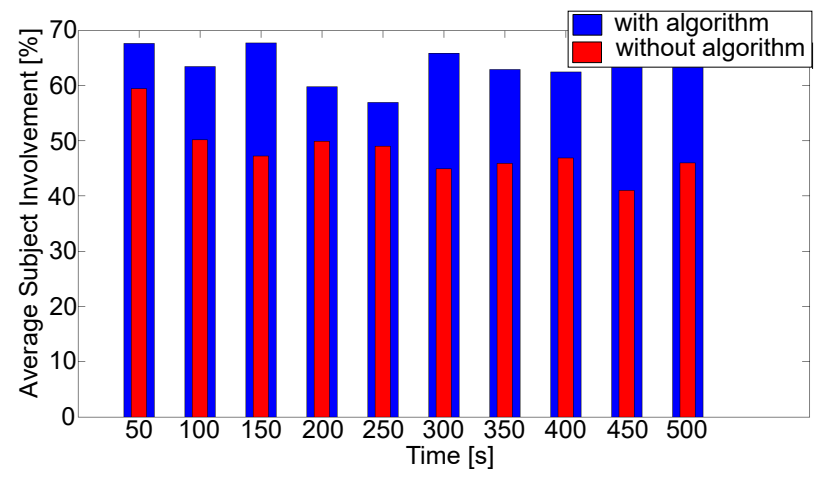

(b)

Fig. 6. The subject involvement performances for the cases with and without the subject adaptive algorithms within the AAN controller averaged for every training block. (a) The averaged subject involvement values for the case with the subject adaptive algorithms resulted consistently higher than the values for the case without the subject adaptive algorithms at every training blocks, except at the training blocks 3 and 8. (b) For the case without the inclusion of the subject adaptive algorithms the average subject involvement shows an apparent decreasing trend as the time progresses. 
trend as the time progresses. This finding supports the claim asserted in [16], which is unless properly challenged, subjects may let the robot take control. On the other hand, for the case with the inclusion of the subject adaptive algorithms, the average subject involvement does not show a decreasing trend, and it is consistently higher than the corresponding cases where the algorithms are not active.

As a further investigation, the average position error and the norm of the control input values are plotted per training block (Fig. 7). As expected, the average position error for the case with the inclusion of the subject adaptive algorithms is higher for every training block (while being less than $2^{\circ}$ for a movement with $20^{\circ}$ amplitude). The finding demonstrates that for the case including the subject adaptive algorithms, the standard deviation of the position error is considerably large. The phenomenon is caused by the fact that the subject adaptive algorithm minimizes the intervention to maintain subject participation. The decrease in the intervention is presented via Fig. 7(b), which shows the norm of the control input. The total control action is consistently smaller in the case where subject adaptive algorithms are active. Combined with the findings presented in the Fig. 5 and 6 , the results in Fig. 7(b) suggest that the subject adaptive algorithms allow more involvement with minimal intervention.

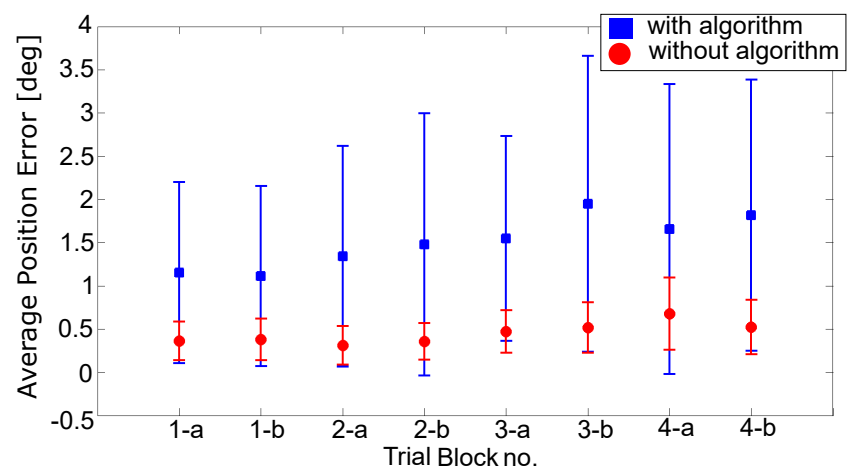

(a)

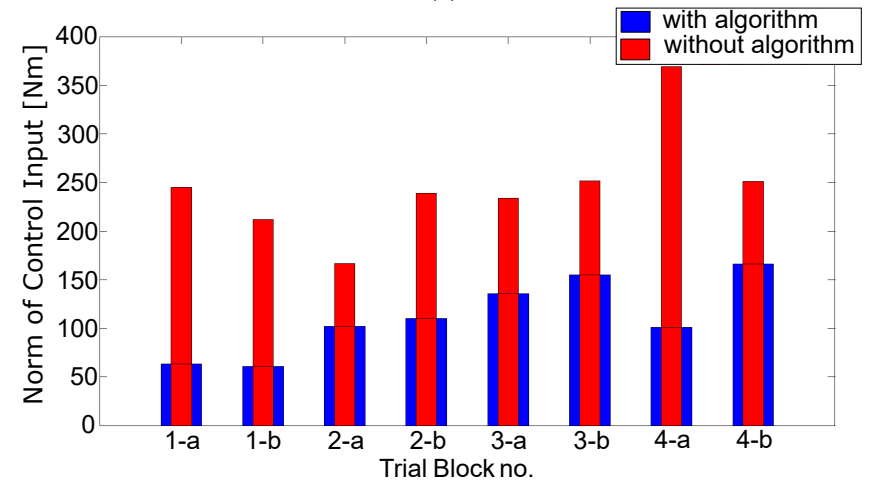

(b)

Fig. 7. The average position error and the norm of the control input values are plotted per training block. (a) The average position error for the case with the inclusion of the subject adaptive algorithms is higher for every training block with considerably larger standard deviation due to the fact that that the subject adaptive algorithms allow the subject to make errors to increase and maintain subject participation. (b) The subject adaptive algorithms minimizes the intervention based on subjects performance.

\section{CONCLUSION}

We presented and experimentally validated a novel AAN controller formulated on a NDO. In addition to the sensorless estimation of subject inputs over time, a bound modification algorithm is introduced, which alters allowable error. Though subject engagement during robotic therapy depends on numerous factors, such as motivation, fatigue, and external distractions, the findings of this pilot study suggest that the inclusion of the subject adaptive algorithms, which challenge the subject based on their performance, maintain subject engagement throughout the robotic rehabilitation sessions.

\section{REFERENCES}

[1] P. S. Lum et al., "Robotic approaches for rehabilitation of hand function after stroke," American J. of Physical Medicine \& Rehabilitation, vol. 91, no. 11, pp. S242-S254, 2012.

[2] Z. Warraich and J. A. Kleim, "Neural plasticity: the biological substrate for neurorehabilitation," $P M \& R$, vol. 2, no. 12, pp. S208-S219, 2010.

[3] A. A. Blank et al., "Current trends in robot-assisted upper-limb stroke rehabilitation: Promoting patient engagement in therapy," Curr. Phys. Med. Rehab. Rep., vol. 2, no. 3, pp. 184-195, 2014.

[4] S. G. Lisberger, "The neural basis for learning of simple motor skills," Science, vol. 242, no. 4879, p. 728, 1988.

[5] J. L. Emken et al., "Robotic movement training as an optimization problem: Designing a controller that assists only as needed," in Rehabilitation Robotics, (ICORR). 9th Intl. Conf. on. IEEE, 2005, pp. 307-312.

[6] H. I. Krebs et al., "Rehabilitation robotics: Performance-based progressive robot-assisted therapy," Autonomous robots, vol. 15, no. 1, pp. 7-20, 2003.

[7] E. T. Wolbrecht et al., "Optimizing compliant, model-based robotic assistance to promote neurorehabilitation," IEEE Trans. on Neural Systems and Rehabilitation Eng, vol. 16, no. 3, pp. 286-297, 2008.

[8] A. U. Pehlivan et al., "A subject-adaptive controller for wrist robotic rehabilitation," IEEE/ASME Transactions on Mechatronics, vol. 20, no. 3, pp. 1338-1350, 2015.

[9] — "Minimal assist-as-needed controller for upper limb robotic rehabilitation," IEEE Transactions on Robotics, vol. 32, no. 1, pp. 113-124, 2016.

[10] W.-H. Chen et al., "A nonlinear disturbance observer for robotic manipulators," IEEE Transactions on industrial Electronics, vol. 47, no. 4, pp. 932-938, 2000.

[11] A. U. Pehlivan et al., "Design and validation of the ricewrist-s exoskeleton for robotic rehabilitation after incomplete spinal cord injury," Robotica, vol. 32, no. 08, pp. 1415-1431, 2014.

[12] A. Gupta and M. K. OMalley, "Disturbance-observer-based force estimation for haptic feedback," Journal of Dynamic Systems, Measurement, and Control, vol. 133, no. 1, p. 014505, 2011.

[13] H. K. Khalil and J. Grizzle, Nonlinear Systems. Upper Saddle River, NJ, USA: Prentice Hall, 2002, vol. 3.

[14] J.-J. E. Slotine and W. Li, "On the adaptive control of robot manipulators," Int. J. of Robotics Research, vol. 6, no. 3, pp. 49-59, 1987.

[15] M. W. Spong et al., Robot Modeling and Control. New York, NY, USA: Wiley, 2006, vol. 3.

[16] E. T. Wolbrecht, "Adaptive, assist-as-needed control of a pneumatic orthosis for optimizing robotic movement therapy following stroke," Ph.D. dissertation, University of California, Irvine, 2007.

[17] C. Krishnan et al., "A pilot study on the feasibility of robot-aided leg motor training to facilitate active participation," PloS one, vol. 8, no. 10, p. e77370, 2013.

[18] P. Konrad, "The ABC of EMG," A Practical Introduction to Kinesiological Electromyography, vol. 1, pp. 30-35, 2005. 